\title{
Tideglusib mitigates dystrophic pathology in skeletal muscle and restores diastolic function
} in young D2 $m d x$ mice

Sophie I. Hamstra ${ }^{\# 1,2}$, Kennedy C. Whitley ${ }^{\# 1,2}$, Jessica L. Braun ${ }^{1,2}$, Briana Hockey ${ }^{1,2}$, Sebastian Silvera $^{1,2}$, Ryan W. Baranowski ${ }^{1,2}$, Emily N. Copeland ${ }^{1,2}$, Mia S. Geromella ${ }^{1,2}$, Colton J.F. Watson $^{3}$, Riley E.G. Cleverdon ${ }^{1,2}$, Rene Vandenboom ${ }^{1,2}$, Brian D. Roy ${ }^{1,2}$, Adam J. MacNeil ${ }^{3}$, Rebecca E.K. MacPherson ${ }^{3}$, Val A. Fajardo ${ }^{1,2^{*}}$

\# These authors contributed equally

${ }^{1}$ Department of Kinesiology, Brock University, St. Catharines, ON, Canada

${ }^{2}$ Centre for Bone and Muscle Health, Brock University, St. Catharines, ON, Canada

${ }^{3}$ Department of Health Sciences, Brock University, St. Catharines, ON, Canada

${ }^{4}$ Department of Kinesiology, Brock University, St. Catharines, ON, Canada

*Corresponding author:

Val A. Fajardo, PhD

Assistant Professor

Canada Research Chair (Tier II)

Department of Kinesiology

Brock University

St. Catharines, ON

L2S 3A1

vfajardo@,brocku.ca 


\begin{abstract}
(150 words)
Duchenne muscular dystrophy (DMD) is a severe muscle wasting disorder that leads to early mortality. We examined the pathogenic contribution of glycogen synthase kinase 3 (GSK3) to DMD using the $m d x$ model. GSK3 is a serine/threonine kinase that has been implicated in other muscular dystrophies and our initial results showed that overactivation of GSK3 may contribute to increased disease severity found in DBA/2J (D2) $m d x$ mice vs C57BL/10 $m d x$ mice. In support of this, treating D2 $m d x$ mice with the GSK3 inhibitor, tideglusib (10 mg/kg/day), increased muscle mass, strength, and fatigue resistance. We also found elevated proportions of oxidative fibers and increased utrophin mRNA, while muscle necrosis and oxidative stress were reduced. Finally, young D2 $m d x$ mice displayed early diastolic dysfunction, and this was blunted with tideglusib treatment - an effect attributed to lowered oxidative stress and fibrosis. This study highlights the therapeutic potential of tideglusib and GSK3 inhibition for DMD.
\end{abstract}




\section{Introduction}

Duchenne muscular dystrophy (DMD) is a severe X-linked disorder that affects 1 in 3,500 males worldwide ${ }^{1}$. It is caused by a single point mutation in the dystrophin gene, and there are over 7000 known mutations that lead to $\mathrm{DMD}^{2}$. These mutations all result in the complete absence of functional dystrophin, a structural protein that provides membrane stability to muscle throughout their strenuous contraction-relaxation cycles ${ }^{1}$. Without dystrophin, muscle integrity is compromised leading to progressive muscle wasting and weakness, particularly of type II glycolytic fibers ${ }^{3,4}$; and patients with DMD are often wheelchair-bound by their teenage years ${ }^{5}$. To date, there is no cure for DMD and despite recent advances in disease management, the average lifespan for an individual with DMD ranges from 20-30 years as they unfortunately succumb to cardiorespiratory failure ${ }^{6}$. Thus, exploring the cellular mechanisms that underpin dystrophic pathology is important as it may reveal novel therapeutic interventions.

Glycogen synthase kinase 3 (GSK3) is a serine/threonine (Ser/Thr) kinase that was first identified for its role in regulating glycogen synthase in muscle ${ }^{7}$, but is now known to phosphorylate more than 100 substrates all over the body ${ }^{8}$. There are two constitutively active and paralogous isoforms, GSK $3 \alpha$ and GSK3 3 , with GSK3 $\beta$ being the most expressed and active isoform found within muscle ${ }^{7}$. GSK3 can be inhibited via Ser phosphorylation (Ser21 on GSK3 $\alpha$ and Ser9 on GSK3 $\beta$ ), preventing GSK3 from binding with its downstream targets ${ }^{8}$. Importantly, the inhibition of GSK3 has been viewed as a viable therapeutic strategy for many diseases and conditions including, but not limited to, Alzheimer's disease, Parkinson's disease, and diabetes ${ }^{9}$. In the context of muscle, GSK3 is a known negative regulator of muscle mass that specifically suppresses protein synthesis and myoblast fusion while also promoting proteolytic degradation (for review see $\operatorname{ref}^{10}$ ). In fact, GSK3 been implicated in other muscle wasting diseases such as 
myotonic dystrophy type 1 (DM1) and limb girdle muscular dystrophy, with its inhibition leading to disease attenuation and enhanced muscle performance ${ }^{11,12}$. However, whether GSK3 plays a pathogenic role in DMD has not yet been fully investigated.

A previous study in C57BL/10 (C57) $m d x$ mice, the most widely used murine model for $\mathrm{DMD}^{13,14}$, found elevated GSK3 expression and activity compared with wild-type (WT) mice ${ }^{15}$. Though this may suggest that GSK3 may contribute to DMD pathology, there has been no study examining the effects of inhibiting GSK3 in $m d x$ mice. Furthermore, it is well-known that the C57 $m d x$ mouse is limited in its capacity to recapitulate DMD as disease pathology in humans is far more severe than that found in these mice, apart from some select muscles (i.e., diaphragm). In 2016, Coley and colleagues ${ }^{16}$ reported a worsened dystrophic phenotype in $m d x$ mice that were backcrossed onto a DBA/2J (D2) strain compared with the C57 strain. With early onset muscle pathology and weakness ${ }^{16-18}$, the D2 $m d x$ mouse has emerged as a viable preclinical model for $\mathrm{DMD}^{17-22}$. In this study, we first compared GSK3 $\beta$ content and activation status (Ser9 phosphorylation) in muscles from age-matched C57 and D2 $m d x$ mice. Using tideglusib, the most clinically advanced GSK3 inhibitor ${ }^{23}$, we then examined the effects of GSK3 inhibition on skeletal and cardiac muscle form and function in young D2 $m d x$ mice.

\section{Results}

GSK3 $\beta$ is more active in D2 mice vs C57 mice and tideglusib treatment reduces GSK3 activation

We compared GSK3 $\beta$ levels in extensor digitorum longus (EDL) muscles across young (910 week old) C57 and D2 WT and $m d x$ mice (Fig. 1a and b). This muscle was chosen at this age, as previous studies have shown muscle weakness and wasting in the D2 $m d x$ EDL but not C57 $m d x \mathrm{EDL}^{16,18}$. Our results show a trending main effect of background strain, where D2 mice, 
irrespective of being WT or $m d x$, had elevated GSK3 $\beta$. When examining Ser9 phosphorylation status of GSK3 3 , we found a significant main effect of background strain, where D2 mice had less Ser9 phosphorylation (relative to total) compared with C57 mice (Fig. 1a and c). These results suggest that D2 mice, both WT and $m d x$, had more active GSK3 in their EDL muscles compared with C57 mice.

To assess the contribution of GSK3 to the dystrophic pathology in D2 $m d x$ mice, we treated them with tideglusib, a non-ATP-competitive GSK3 inhibitor and member of the thidiazolidinone family (TDZD). We treated these mice with a dose of $10 \mathrm{mg} / \mathrm{kg} /$ day via oral gavage for $2-4$ weeks (5 days per week) starting at 6-7 weeks of age. Our results show that tideglusib significantly lowered GSK3 $\beta$ after 2 and 4 weeks of treatment (Fig. 1d and Supplemental Fig.1). Further, with 4 weeks of treatment, tideglusib increased the ratio of Ser9 phosphorylation to total GSK $3 \beta$ content in the EDL muscles from D2 $m d x$ mice compared with vehicle-treated D2 $m d x$ mice (Fig. $1 \mathrm{~d}$ ). $\beta$ catenin serves as a downstream marker of GSK3 activation since GSK3 can phosphorylate $\beta$ catenin leading to its proteolytic degradation ${ }^{24}$. Not surprisingly, we saw a significant increase in $\beta$-catenin with tideglusib treatment in the D2 $m d x$ EDL (Fig. 1e).

Tideglusib increases muscle mass and oxidative fiber type content while reducing muscle necrosis Consistent with its role as a negative regulator of muscle size ${ }^{10}$, we found that GSK3 inhibition with tideglusib treatment restored EDL:body mass ratio in D2 $m d x$ mice to levels comparable with WT mice (Fig. 2a). Furthermore, longitudinal analyses of body composition using a small animal DXA suggests that D2 $m d x$ mice treated with tideglusib experienced the largest gains in lean mass throughout the 4 week treatment period compared with vehicle treated D2 $m d x$ mice; ableit, this did not reach statistical significance (Fig. 2b). On average, $m d x$-vehicle 
treated mice gained 3.0. \pm 0.4 g of body mass throughout the 4-week treatment protocol, whereas $m d x$-vehicle treated only gained $2.1 \pm 0.3 \mathrm{~g}$ (Student's t-test, $p=0.11$ ). In addition to regulating muscle size, GSK3 also represses the oxidative myogenic program ${ }^{10,25}$. Skeletal muscle fibers can be classified as either oxidative (I and IIA) or glycolytic (IIX and IIB) and are distinguished by their contractile kinetics, fatigue susceptibility, and metabolic machinery. In the context of DMD, muscle fiber types can also be differentiated by their susceptibility to dystrophic pathology, with oxidative muscle fiber types (I and IIA) being more resistant than glycolytic fibers. This is in large part owed to their relative greater expression of utrophin - a dystrophin homolog that can compensate for the absence of dystrophin ${ }^{4}$. Here, we found that tideglusib treatment enhanced the oxidative myogenic program in D2 $m d x$ mice, leading to an increased percentage of oxidative (I and IIA) fibers and utrophin mRNA levels (Fig. 2c-e). In turn, histological analyses showed significant reductions in necrosis in EDL muscles from tideglusib treated D2 $m d x$ mice compared with vehicle treated D2 $m d x$ mice (Figure $2 \mathrm{f}$ and $\mathrm{g}$ ). Measuring serum creatine kinase (CK) levels as a marker of muscle damage, we found that serum CK was significantly elevated in vehicle treated D2 $m d x$ mice when compared with WT mice (Fig. 2h). However, tideglusib treatment blunted this effect as serum CK was significantly lower in tideglusib treated D2 mdx mice vs vehicle treated D2 $m d x$ mice (Fig. 2h). Finally, we conducted western blot analysis of total nitrotyrosine and nitrocysteine levels as indicators of oxidative stress. Our results show that vehicle treated D2 $m d x$ EDL muscles had the highest levels of oxidative stress, which was lowered with tideglusib treatment; albeit this was only significant for total nitrotyrosine and not nitrocysteine (Fig. 2i). 


\section{GSK3 inhibition with tideglusib improves whole-body and muscle performance}

From a functional standpoint, vehicle treated D2 $m d x$ mice were less active in their cages compared with WT mice; however, tideglusib treatment blunted this effect as cage activity in tideglusib treated D2 $m d x$ mice was not significantly different from WT mice (Fig. 3a). Similarly, hangwire tests revealed that vehicle treated D2 $m d x$ mice had less impulse generated (seconds on the wire $\mathrm{x}$ body mass of mouse) compared with WT mice, which was also attenuated with tideglusib treatment (Fig. 3b). These results indicate that tideglusib treatment enhanced wholebody muscle performance and fatigue resistance in D2 $m d x$ mice; and corresponding well with this, tideglusib treatment enhanced fatigue resistance in isolated EDL muscles (Fig. 3c). Muscles from vehicle treated D2 $m d x$ mice took less time to reach $50 \%$ of initial force when applied to a fatigue protocol $(70 \mathrm{~Hz}$ every $2 \mathrm{~s}$ for $5 \mathrm{~min}$; Fig. 3c); however, this effect was blunted in D2 $m d x$ mice treated with tideglusib. Finally, force-frequency analyses of isolated EDL muscles showed that while WT mice produced greater specific isometric force $\left(\mathrm{mN} / \mathrm{mm}^{2}\right)$ compared with both tideglusib and vehicle treated D2 $m d x$ mice, tideglusib treated $m d x$ mice also significantly produced more force compared with vehicle treated mice, particularly at stimulation frequencies of 100 and $150 \mathrm{~Hz}$ (Fig. 3d).

\section{Concentric remodelling and diastolic dysfunction in young D2 mdx mice}

Table 1 shows the structural and functional analyses in WT, vehicle- and tideglusib treated D2 $m d x$ mice. There were no differences in measured left ventricle (LV) mass relative to body mass. With high frequency ultrasound, we found signs of concentric remodelling with lowered left ventricle internal diameter (LVID) and increased relative wall thickness (RWT). Both enddiastolic volume (EDV) and end-systolic volume (ESV) were lowered in vehicle treated and 
tideglusib treated D2 $m d x$ mice compared with WT, however, this was only statistically significant for ESV. Despite the reduction in ESV, there were no changes in stroke volume (SV) across groups, which in turn led to significantly higher ejection fraction (EF) in vehicle treated and tideglusib treated D2 $m d x$ mice compared with WT. Interestingly, when examining isovolumic contraction and relaxation times (IVCT and IVRT, respectively), we found that IVRT was significantly highest in the vehicle treated D2 $m d x$ mice compared with both WT and tideglusib treated D2 $m d x$ mice. Overall, these results indicate that young D2 $m d x$ mice show signs of concentric remodelling; and while systolic function is intact, and apparently even enhanced, diastolic dysfunction can be observed in young vehicle treated D2 $m d x$ mice, but not age-matched tideglusib treated D2 $m d x$ mice.

Tideglusib treatment reduces cardiac GSK3, oxidative stress and fibrosis in D2 mdx mice

To understand the mechanisms behind the apparent improvements in diastolic function in tideglusib treated D2 $m d x$ mice, we first assessed LV sarco(endo)plasmic reticulum $\mathrm{Ca}^{2+}$-ATPase (SERCA) function by measuring rates of $\mathrm{Ca}^{2+}$ uptake and ATPase activity. SERCA catalyzes the active transport of $\mathrm{Ca}^{2+}$ ions into the sarcoplasmic reticulum (SR) thereby eliciting muscle relaxation. Similar to our results in EDL muscle, we saw a reduction in total GSK3 $\beta$ content in tideglusib treated vs. vehicle treated $m d x$ mice (Fig. 4a and b). Another result, similar to our recent observations (published preprint ${ }^{26}$ and currently under review), shows that D2 $m d x$ mice (vehicle and tideglusib treated) had significant impairments in $\mathrm{Ca}^{2+}$ uptake (Fig. 4c and d). Furthermore, maximal SERCA activity appeared to be lower in both vehicle and tideglusib treated D2 $m d x$ mice compared with WT, however, this was not statistically significant (Fig. 4e). There were no 
differences in SERCA's apparent affinity for $\mathrm{Ca}^{2+}$ across groups, nor were there differences in the protein levels of SERCA2 or its regulator phospholamban (PLN) (Fig. 4f-h).

In the absence of any major improvement in SERCA function and $\mathrm{Ca}^{2+}$ handling with tideglusib treatment, we next examined levels of oxidative stress - given its implication in stiffening the myocardium ${ }^{27,28}$. Similar to the EDL muscle, western blot analysis showed that levels of total nitrotyrosine and nitrocysteine were highest in vehicle treated D2 $m d x$ mice, but then were lowered with tideglusib treatment (Fig. $4 \mathrm{i}$ and $\mathrm{j}$ ). This was only statistically significant for total nitrocysteine. In the diaphragm, a severely affected muscle across $m d x$ mouse models, we found similar results with the LV, where there were no major improvements in SERCA function assessed through $\mathrm{Ca}^{2+}$ uptake and SERCA activity with tideglusib treatment; however, tideglusib blunted the increase in nitrotyrosine and nitrocysteine seen in vehicle treated mdx mice (Supplementary Fig. 2).

Finally, we measured fibrosis using Gamori Trichrome staining in the LV. Fig. 5 shows that vehicle treated D2 $m d x$ mice had mostly higher levels of fibrosis across the apex and the base. Notable variability was observed in vehicle treated D2 $m d x$ hearts, which is consistent with a previous study using these mice at a similar age ${ }^{17}$. Though this has limited our statistical analysis, we did find that tideglusib treated D2 $m d x$ hearts tended to have less fibrosis compared with vehicle treated $m d x$ hearts (Fig. 5).

\section{Discussion}

Here, we sought to investigate the role of GSK3 in dystrophic pathology in the $m d x$ mouse model for DMD. In the first part of this study, we found that D2 mice (WT and $m d x$ ) had greater levels of GSK3 activation in their EDL muscles compared with those from age-matched C57 mice. 
While we did not detect a main effect of $m d x$ genotype on elevating GSK3 content and activation status relative to WT control, we believe that the apparent strain difference in GSK3 activation status likely contributes to the well-established differences in disease pathology between the less severe C57 $m d x$ mouse and the more severe D2 $m d x$ mouse $^{16-18}$. In support of this, treating D2 $m d x$ mice with the GSK3 inhibitor, tideglusib, increased EDL muscle mass, the proportion of oxidative fibers and utrophin expression, while also reducing muscle necrosis and oxidative stress. This ultimately improved whole-body and isolated muscle performance and fatigue resistance. Furthermore in cardiac muscle, tideglusib treatment attenuated the early diastolic dysfunction observed in vehicle treated D2 $m d x$ mice by lowering LV fibrosis and oxidative stress.

Tideglusib is the most clinically advanced GSK3 inhibitor that is currently being used in Phase II clinical trials for Alzheimer's disease, progressive supranuclear palsy, myotonic dystrophy, and autism spectrum disorders ${ }^{23,29}$. We view this to be a strength of our study as examining a GSK3 inhibitor that is already in clinical trials for other diseases could lead to a more rapid clinical integration for DMD. Moreover, the chosen dose for this study is in line with those used for clinical trials for myotonic dystrophy $(400-1000 \mathrm{mg}$ per day for patients aged 15-34 years) and shown to be well tolerated and safe ${ }^{30}$. Across both skeletal and cardiac muscles, we found that tideglusib treatment lowered total GSK3 $\beta$ content. This finding is similar to that of a previous study that treated a preclinical DM1 mouse model with another TDZD class drug ${ }^{11}$. In this study, Jones and colleagues found that two days of TDZD- 8 treatment lowered GSK3 $\beta$ content via a reduction in tyrosine 216 phosphorylation, which is thought to stabilize GSK3 $\beta$. We also found that tideglusib treatment increased the level of inhibitory Ser9 phosphorylation of GSK3 $\beta$ in the EDL and the LV (non-significantly), however, it is important to note that outside of changes 
in total and phosphorylated GSK3 $\beta$, tideglusib's purported inhibitory mechanism of action is to stabilize GSK3 in an inactive configuration ${ }^{31}$.

With tideglusib treatment we found a restoration in EDL:body mass ratio and significantly larger gains in absolute lean muscle mass in D2 $m d x$ mice. This result is largely consistent with the role of GSK3 in negatively regulating muscle mass, which can occur through a number of pathways including an acceleration of muscle proteolysis, inhibition of muscle protein synthesis, and an attenuation of myoblast fusion ${ }^{10,32-34}$. The latter is very important in the context of DMD, which is characterized by constant degeneration-regeneration cycling. Indeed, GSK3 inhibition has been shown to accelerate muscle regeneration ${ }^{35}$. One pathway largely implicated in myoblast fusion and regeneration is the calcineurin pathway ${ }^{36-39}$. Calcineurin is a $\mathrm{Ca}^{2+}$-dependent phosphatase that dephosphorylates nuclear factor of activated T-cell (NFAT) transcription factors that in muscle control the transcription of genes associated with muscle development, growth, and the oxidative fiber type $\mathrm{e}^{40,41}$. It is well-established that GSK3 counteracts calcineurin by rephosphorylating NFAT, thereby preventing its nuclear activation and the transcription of its downstream targets ${ }^{10}$. Thus, in addition to its role in regulating muscle mass, we and others have shown that GSK3 negatively regulates the oxidative fiber type, and that its inhibition in muscle can promote the oxidative myogenic program ${ }^{10,25,42}$. Our findings presented here are strongly aligned with this, as we observed a significant increase in the proportion of oxidative (I and IIA) fibers in tideglusib treated vs vehicle treated D2 $m d x$ EDL muscles. This is important as these fibers are known to be more resistant to dystrophic pathology, partly because of their increased utrophin expression ${ }^{4}$. It has been well established that utrophin can counteract dystrophic pathology in $m d x$ mice, with an increase in its expression attenuating disease in $C 57^{3,43-45}$ and D2 ${ }^{46}$ $m d x$ mice; and its genetic deletion accelerating disease in C57 $m d x$ mice ${ }^{47,48}$. Furthermore, since 
utrophin is largely regulated by calcineurin signalling ${ }^{3,4}$ our observations of increased utrophin expression with tideglusib treatment in D2 $m d x$ skeletal muscle further showcases the repressive action of GSK3 on calcineurin.

The increased proportion of oxidative fibers and utrophin expression contributed to the enhancement in muscle quality in the D2 $m d x$ mice. This was evident through a reduction in serum CK levels, muscle necrosis and oxidative stress. Together, the improvements in muscle mass and quality enhanced whole-body muscle performance measures of cage ambulation and hangwire impulse, as well as specific force and fatigue resistance assessed in isolated EDL muscles. The enhancement in fatigue resistance is not surprising given that oxidative fibers are relatively more fatigue resistant compared with their glycolytic counterparts. Collectively, our findings clearly demonstrate that short-term tideglusib treatment improves skeletal muscle form and function in the D2 $m d x$ mouse.

In addition to skeletal muscle, we also examined the effects of tideglusib treatment on cardiac muscle form and function since patients with DMD often succumb to cardiorespiratory failure. While fibrosis and oxidative stress have been observed in young (8-12 week old) D2 $m d x$ mice, cardiac contractile deficits, namely a reduction in EF\% was only found in older mice $(\sim 28$ weeks $)^{16}$. Here, we did not find any deficits in EF\%, in fact we observed the opposite, where D2 $m d x$ mice (vehicle and tideglusib treated) had greater EF\% compared with WT controls. This apparent increase in systolic function is consistent with a previous study using 21-week-old D2 $m d x$ mice $^{46}$. In contrast with systolic function, our study is the first to show signs of diastolic dysfunction (prolonged IVRT) in young vehicle treated D2 $m d x$ mice. Therefore, diastolic dysfunction may be an early event that precedes any impairments in EF and cardiac contractility that, more importantly, can be blunted with tideglusib treatment. 
Several factors can contribute to the observed diastolic dysfunction found in vehicle treated D2 $m d x$ mice including concentric remodelling (reduced LVID;s and increased RWT;s), impairments in SERCA function and reduced compliance found with elevated fibrosis and oxidative stress. Of note, the reduction in LVID can also contribute to the apparent increase in EF by reducing EDV, though non-significantly, in the face of preserved SV. SERCA is a $\mathrm{Ca}^{2+}$ regulatory protein that is responsible for at least $70 \%$ of cytosolic $\mathrm{Ca}^{2+}$ removal in the heart making it a critical player in cardiac muscle relaxation and diastolic function. Indeed, its impairment has been largely implicated in DMD pathology ${ }^{49-52}$. We and others have shown that GSK3 negatively regulates SERCA by reducing its expression ${ }^{53-55}$; however, tideglusib treatment did not restore SERCA function in the D2 $m d x \mathrm{LV}$ or diaphragm. Fibrosis and the ensuing reduction in compliance can add to myocardial stiffening which can also negatively affect diastolic function ${ }^{28}$. Here, we saw a reduction in LV fibrosis in tideglusib treated D2 $m d x$ mice vs vehicle treated D2 $m d x$ mice which we speculate could contribute to the improvements in diastolic function and IVRT observed in tideglusib treated D2 $m d x$ mice, though this only trended towards statistical significance. Finally, elevated oxidative stress can also lead to diastolic dysfunction, purportedly through negative alterations in titin which increase passive stiffness in the cardiomyocyte ${ }^{28}$. Across the D2 $m d x$ LV, EDL and diaphragm, we found that tideglusib blunted oxidative stress (lowered nitrotyrosine and/or nitrocysteine). This result is in line with previous reports showing that GSK3 inhibition attenuates oxidative stress associated with ischemia-reperfusion models ${ }^{56-58}$. Moreover, in ischemic and non-ischemic myocardial tissue, GSK3 inhibition reduced collagen deposition and oxidative stress ${ }^{57}$, which is consistent with our findings here in D2 $m d x$ mice.

It should also be noted that although active GSK3 is meant to have cardiac antihypertrophic functions ${ }^{59}$, tideglusib-mediated inhibition of GSK3 did not significantly alter 
LV:body weight mass. This, combined with improvements in diastolic function, suggest that inhibiting cardiac GSK3 could be an effective therapeutic strategy in the treatment and prevention of cardiomyopathies which are a common result of this disease ${ }^{16,17}$. In fact, GSK3 inhibition has already been targeted in a preclinical model of arrhythmogenic cardiomyopathy (the $D s g 2^{\text {mut } / \text { mut }}$ mouse) and has shown similar improvements in cardiac function and fibrotic remodelling ${ }^{60}$. Altogether, these findings indicate that the early diastolic dysfunction observed in young D2 $m d x$ mice can be restored with tideglusib treatment by a reduction in fibrosis and oxidative stress.

In summary, our study shows that short-term GSK3 inhibition via tideglusib treatment in D2 $m d x$ mice improves skeletal and cardiac muscle form and function by acting on several pathways and reducing muscle necrosis, fibrosis, and oxidative stress. Future studies should examine the longer-term effects of tideglusib treatment and/or GSK3 inhibition to determine whether GSK3 inhibition can improve the quantity and quality of life in these mice. As tideglusib is the most clinically advanced GSK3 inhibitor known to date, future clinical studies investigating whether this TDZD drug can be repurposed for DMD should also be performed. 
bioRxiv preprint doi: https://doi.org/10.1101/2022.02.16.480726; this version posted February 16,2022 . The copyright holder for this preprint (which was not certified by peer review) is the author/funder. All rights reserved. No reuse allowed without permission. 


\begin{abstract}
Author contributions
$\mathrm{SIH}$ - Concept design, writing and drafting the manuscript, figure creation, data collection, data interpretation

KCW - Concept design, writing and drafting the manuscript, figure creation, data collection, data interpretation

JLB - Data collection, data interpretation, reviewing and revising the manuscript

$\mathrm{BH}$ - Data collection, data interpretation, reviewing and revising the manuscript

SS - Data collection, data interpretation, reviewing and revising the manuscript

RWB - Data collection, data interpretation, reviewing and revising the manuscript

EC - Data collection, data interpretation, reviewing and revising the manuscript

MG - Data collection, data interpretation, reviewing and revising the manuscript

CJFW - Data collection, data interpretation, reviewing and revising the manuscript

REGC - Data collection, data interpretation, reviewing and revising the manuscript

RV - Provision of reagents and resources, reviewing and revising the manuscript

BDR - Provision of reagents and resources, reviewing and revising the manuscript

AJM - Provision of reagents and resources, reviewing and revising the manuscript

REKM - Provision of reagents and resources, reviewing and revising the manuscript

VAF - Concept design, supervision, provision of reagents and resources, acquiring funding, writing and drafting the manuscript
\end{abstract}

\title{
Acknowledgements
}

This work was supported by an unrestricted Brock University Grant and a Canada Research Chair

(Tier 2) award to VAF. SIH was supported by a NSERC CGS-M, and JLB was supported by a

CIHR CGS-M. We thank Tonya Coulthard (Scintica Instruments) for her help with high frequency ultrasound analysis.

\section{Conflict of interest}

The authors declare that there are no conflicts of interests to report. 


\section{References}

1. Houang, E.M., Sham, Y.Y., Bates, F.S. \& Metzger, J.M. Muscle membrane integrity in Duchenne muscular dystrophy: recent advances in copolymer-based muscle membrane stabilizers. Skelet Muscle 8, 31 (2018).

2. Bladen, C.L., et al. The TREAT-NMD DMD Global Database: analysis of more than 7,000 Duchenne muscular dystrophy mutations. Hum Mutat 36, 395-402 (2015).

3. Chakkalakal, J.V., et al. Stimulation of calcineurin signaling attenuates the dystrophic pathology in mdx mice. Hum Mol Genet 13, 379-388 (2004).

4. Chakkalakal, J.V., et al. Expression of utrophin A mRNA correlates with the oxidative capacity of skeletal muscle fiber types and is regulated by calcineurin/NFAT signaling. Proc Natl Acad Sci U S A 100, 7791-7796 (2003).

5. Song, T.J., Lee, K.A., Kang, S.W., Cho, H. \& Choi, Y.C. Three cases of manifesting female carriers in patients with Duchenne muscular dystrophy. Yonsei Med J 52, 192-195 (2011).

6. Laviola, M., Priori, R., D'Angelo, M.G. \& Aliverti, A. Assessment of diaphragmatic thickness by ultrasonography in Duchenne muscular dystrophy (DMD) patients. PLoS One 13, e0200582 (2018).

7. Wang, H., Brown, J. \& Martin, M. Glycogen synthase kinase 3: a point of convergence for the host inflammatory response. Cytokine 53, 130-140 (2011).

8. Beurel, E., Grieco, S.F. \& Jope, R.S. Glycogen synthase kinase-3 (GSK3): regulation, actions, and diseases. Pharmacol Ther 148, 114-131 (2015).

9. Cohen, P. \& Goedert, M. GSK3 inhibitors: development and therapeutic potential. Nat Rev Drug Discov 3, 479-487 (2004).

10. Mirzoev, T.M., Sharlo, K.A. \& Shenkman, B.S. The Role of GSK-3 $\beta$ in the Regulation of Protein Turnover, Myosin Phenotype, and Oxidative Capacity in Skeletal Muscle under Disuse Conditions. Int J Mol Sci 22, 5081 (2021).

11. Jones, K., et al. GSK3beta mediates muscle pathology in myotonic dystrophy. J Clin Invest 122, 4461-4472 (2012).

12. Findlay, A.R., et al. Lithium chloride corrects weakness and myopathology in a preclinical model of LGMD1D. Neurol Genet 5, e318 (2019).

13. Bulfield, G., Siller, W.G., Wight, P.A. \& Moore, K.J. X chromosome-linked muscular dystrophy (mdx) in the mouse. Proc Natl Acad Sci U S A 81, 1189-1192 (1984).

14. Dangain, J. \& Vrbova, G. Muscle development in mdx mutant mice. Muscle Nerve 7, 700-704 (1984).

15. Villa-Moruzzi, E., Puntoni, F. \& Marin, O. Activation of protein phosphatase-1 isoforms and glycogen synthase kinase-3 beta in muscle from mdx mice. Int J Biochem Cell Biol 28, 13-22 (1996).

16. Coley, W.D., et al. Effect of genetic background on the dystrophic phenotype in mdx mice. Hum Mol Genet 25, 130-145 (2016).

17. van Putten, M., et al. Natural disease history of the D2-mdx mouse model for Duchenne muscular dystrophy. FASEB J 33, 8110-8124 (2019).

18. Hammers, D.W., et al. The D2.mdx mouse as a preclinical model of the skeletal muscle pathology associated with Duchenne muscular dystrophy. Sci Rep 10, 14070 (2020).

19. Ramos, S.V., Hughes, M.C., Delfinis, L.J., Bellissimo, C.A. \& Perry, C.G.R. Mitochondrial bioenergetic dysfunction in the D2.mdx model of Duchenne muscular dystrophy is associated with microtubule disorganization in skeletal muscle. PLoS One 15, e0237138 (2020). 
20. Spaulding, H.R., Quindry, T., Quindry, J.C. \& Selsby, J.T. Nutraceutical and pharmaceutical cocktails did not preserve diaphragm muscle function or reduce muscle damage in D2-mdx mice. Exp Physiol 105, 989-999 (2020).

21. Su, X., Shen, Y., Jin, Y., Weintraub, N.L. \& Tang, Y.L. Identification of critical molecular pathways involved in exosome-mediated improvement of cardiac function in a mouse model of muscular dystrophy. Acta Pharmacol Sin (2020).

22. Hammers, D.W., et al. Glucocorticoids counteract hypertrophic effects of myostatin inhibition in dystrophic muscle. JCl Insight 5(2020).

23. Noori, M.S., et al. Identification of a novel selective and potent inhibitor of glycogen synthase kinase-3. Am J Physiol Cell Physiol 317, C1289-c1303 (2019).

24. Wu, D. \& Pan, W. GSK3: a multifaceted kinase in Wnt signaling. Trends Biochem Sci 35, 161-168 (2010).

25. Whitley, K.C., et al. GSK3 inhibition with low dose lithium supplementation augments murine muscle fatigue resistance and specific force production. Physiol Rep 8, e14517 (2020).

26. Cleverdon, R.E., et al. Sarcoplasmic reticulum $\mathrm{Ca}<\mathrm{sup}>2+</$ sup $>$ handling in young $\mathrm{DBA} / 2 \mathrm{~J}$ vs C57BL/10 mdx models of Duchenne muscular dystrophy. bioRxiv, 2021.2010.2025.465805 (2021).

27. Beckendorf, L. \& Linke, W.A. Emerging importance of oxidative stress in regulating striated muscle elasticity. J Muscle Res Cell Motil 36, 25-36 (2015).

28. Zhazykbayeva, S., Pabel, S., Mügge, A., Sossalla, S. \& Hamdani, N. The molecular mechanisms associated with the physiological responses to inflammation and oxidative stress in cardiovascular diseases. Biophys Rev 12, 947-968 (2020).

29. Khan, I., Tantray, M.A., Alam, M.S. \& Hamid, H. Natural and synthetic bioactive inhibitors of glycogen synthase kinase. Eur J Med Chem 125, 464-477 (2017).

30. Horrigan, J., et al. A Phase 2 Study of AMO-02 (Tideglusib) in Congenital and Childhood-Onset Myotonic Dystrophy Type 1 (DM1). Pediatr Neurol 112, 84-93 (2020).

31. Balasubramaniam, M., et al. Structural modeling of GSK3 $\beta$ implicates the inactive (DFG-out) conformation as the target bound by TDZD analogs. Scientific Reports 10, 18326 (2020).

32. Kurgan, N., et al. A Low-Therapeutic Dose of Lithium Inhibits GSK3 and Enhances Myoblast Fusion in C2C12 Cells. Cells 8(2019).

33. Verhees, K.J., et al. Glycogen synthase kinase-3beta is required for the induction of skeletal muscle atrophy. Am J Physiol Cell Physiol 301, C995-C1007 (2011).

34. Rommel, C., et al. Mediation of IGF-1-induced skeletal myotube hypertrophy by $\mathrm{PI}(3) \mathrm{K} / \mathrm{Akt} / \mathrm{mTOR}$ and PI(3)K/Akt/GSK3 pathways. Nat Cell Biol 3, 1009-1013 (2001).

35. Pansters, N.A., et al. Muscle-specific GSK-3beta ablation accelerates regeneration of disuseatrophied skeletal muscle. Biochim Biophys Acta 1852, 490-506 (2015).

36. Pansters, N.A., et al. Segregation of myoblast fusion and muscle-specific gene expression by distinct ligand-dependent inactivation of GSK-3beta. Cell Mol Life Sci 68, 523-535 (2011).

37. Fajardo, V.A., et al. Neurogranin is expressed in mammalian skeletal muscle and inhibits calcineurin signaling and myoblast fusion. Am J Physiol Cell Physiol 317, C1025-C1033 (2019).

38. Park, S.Y., et al. Stabilin-2 modulates the efficiency of myoblast fusion during myogenic differentiation and muscle regeneration. Nat Commun 7, 10871 (2016).

39. Stupka, N., Gregorevic, P., Plant, D.R. \& Lynch, G.S. The calcineurin signal transduction pathway is essential for successful muscle regeneration in $\mathrm{mdx}$ dystrophic mice. Acta Neuropathol 107, 299-310 (2004).

40. Michel, R.N., Dunn, S.E. \& Chin, E.R. Calcineurin and skeletal muscle growth. Proc Nutr Soc 63, 341-349 (2004). 
41. Michel, R.N., Chin, E.R., Chakkalakal, J.V., Eibl, J.K. \& Jasmin, B.J. Ca2+/calmodulin-based signalling in the regulation of the muscle fibre phenotype and its therapeutic potential via modulation of utrophin A and myostatin expression. Appl Physiol Nutr Metab 32, 921-929 (2007).

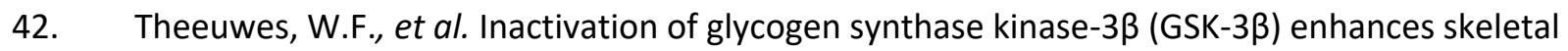
muscle oxidative metabolism. Biochim Biophys Acta Mol Basis Dis 1863, 3075-3086 (2017).

43. Tinsley, J., et al. Expression of full-length utrophin prevents muscular dystrophy in $\mathrm{mdx}$ mice. Nat Med 4, 1441-1444 (1998).

44. Squire, S., et al. Prevention of pathology in $\mathrm{mdx}$ mice by expression of utrophin: analysis using an inducible transgenic expression system. Hum Mol Genet 11, 3333-3344 (2002).

45. Fajardo, V.A., et al. Sarcolipin deletion in mdx mice impairs calcineurin signalling and worsens dystrophic pathology. Hum Mol Genet (2018).

46. Kennedy, T.L., et al. Micro-utrophin Improves Cardiac and Skeletal Muscle Function of Severely Affected D2/mdx Mice. Mol Ther Methods Clin Dev 11, 92-105 (2018).

47. Deconinck, A.E., et al. Utrophin-dystrophin-deficient mice as a model for Duchenne muscular dystrophy. Cell 90, 717-727 (1997).

48. Grady, R.M., et al. Skeletal and cardiac myopathies in mice lacking utrophin and dystrophin: a model for Duchenne muscular dystrophy. Cell 90, 729-738 (1997).

49. Morine, K.J., Sleeper, M.M., Barton, E.R. \& Sweeney, H.L. Overexpression of SERCA1a in the $\mathrm{mdx}$ diaphragm reduces susceptibility to contraction-induced damage. Hum Gene Ther 21, 1735-1739 (2010).

50. Goonasekera, S.A., et al. Mitigation of muscular dystrophy in mice by SERCA overexpression in skeletal muscle. J Clin Invest 121, 1044-1052 (2011).

51. Mazala, D.A., et al. SERCA1 overexpression minimizes skeletal muscle damage in dystrophic mouse models. Am J Physiol Cell Physiol 308, C699-709 (2015).

52. Gehrig, S.M., et al. Hsp72 preserves muscle function and slows progression of severe muscular dystrophy. Nature 484, 394-398 (2012).

53. Hamstra, S.I., et al. Low-dose lithium feeding increases the SERCA2a-to-phospholamban ratio, improving SERCA function in murine left ventricles. Exp Physiol 105, 666-675 (2020).

54. Hamstra, S.I., et al. The role of phospholamban and GSK3 in regulating rodent cardiac SERCA function. Am J Physiol Cell Physiol 319, C694-c699 (2020).

55. Michael, A., et al. Glycogen synthase kinase-3beta regulates growth, calcium homeostasis, and diastolic function in the heart. J Biol Chem 279, 21383-21393 (2004).

56. Rana, A.K. \& Singh, D. Targeting glycogen synthase kinase-3 for oxidative stress and neuroinflammation: Opportunities, challenges and future directions for cerebral stroke management. Neuropharmacology 139, 124-136 (2018).

57. Potz, B.A., et al. Glycogen synthase kinase $3 \beta$ inhibition reduces mitochondrial oxidative stress in chronic myocardial ischemia. J Thorac Cardiovasc Surg 155, 2492-2503 (2018).

58. Singh, S.P., et al. Glycogen synthase kinase-3 inhibition attenuates fibroblast activation and development of fibrosis following renal ischemia-reperfusion in mice. Dis Model Mech 8, 931940 (2015).

59. Hardt, S.E. \& Sadoshima, J. Glycogen synthase kinase-3ß: a novel regulator of cardiac hypertrophy and development. Circulation research 90, 1055-1063 (2002).

60. Chelko, S.P., et al. Central role for GSK3beta in the pathogenesis of arrhythmogenic cardiomyopathy. JCI Insight 1(2016).

61. Whitley, K.C., et al. GSK3 inhibition with low dose lithium supplementation augments murine muscle fatigue resistance and specific force production. Physiological Reports (2020).

62. Mendez, J.K., A. Density and compositon of mammalian muscle. Metabolism 9, 184-188 (1960). 
63. Brooks, S.V. \& Faulkner, J.A. Contractile properties of skeletal muscles from young, adult and aged mice. Journal of Physiology 404, 71-82 (1988).

64. Fajardo, V.A., et al. Diaphragm assessment in mice overexpressing phospholamban in slowtwitch type I muscle fibers. Brain Behav 6, e00470 (2016).

65. Bloemberg, D. \& Quadrilatero, J. Rapid determination of myosin heavy chain expression in rat, mouse, and human skeletal muscle using multicolor immunofluorescence analysis. PLoS One 7, e35273 (2012).

66. Hamstra, S.I., et al. Low-dose lithium feeding increases the SERCA2a to phospholamban ratio improving SERCA function in murine left ventricles. Exp Physiol (2020).

67. Braun, J.L., Geromella, M.S., Hamstra, S.I., Messner, H.N. \& Fajardo, V.A. Characterizing SERCA Function in Murine Skeletal Muscles after 35-37 Days of Spaceflight. Int J Mol Sci 22(2021).

68. Braun, J.L., et al. Neuronatin promotes SERCA uncoupling and its expression is altered in skeletal muscles of high-fat diet-fed mice. FEBS Lett 595, 2756-2767 (2021). 
Table 1. High-frequency ultrasound analysis in young age-matched wild-type (WT), vehicle and tideglusib treated $m d x$ mice.

\begin{tabular}{lccc}
\hline & WT & mdx-vehicle & md $\boldsymbol{x}$-tideglusib \\
\hline LV:body mass & $3.81 \pm 0.12$ & $3.81 \pm 0.15$ & $4.0 \pm 0.14$ \\
LVID;d (mm) & $3.59 \pm 0.12$ & $3.29 \pm 0.16$ & $3.31 \pm 0.13$ \\
LVID;s (mm) & $2.33 \pm 0.10$ & $1.85 \pm 0.10^{* *}$ & $1.82 \pm 0.13^{* *}$ \\
RWT;d & $0.58 \pm 0.03$ & $0.69 \pm 0.04$ & $0.71 \pm 0.04$ \\
RWT;s & $1.23 \pm 0.08$ & $1.81 \pm 0.15^{*}$ & $1.88 \pm 0.16^{* *}$ \\
& & & \\
EDV (ml) & $55.10 \pm 4.11$ & $44.52 \pm 3.91$ & $42.00 \pm 2.90$ \\
ESV (ml) & $19.32 \pm 1.89$ & $11.71 \pm 1.59^{* *}$ & $10.85 \pm 2.00^{* *}$ \\
SV (ml) & $35.78 \pm 2.84$ & $33.35 \pm 2.5$ & $34.5 \pm 2.8$ \\
EF (\%) & $65.0 \pm 2.2$ & $75.7 \pm 1.5^{* *}$ & $77.0 \pm 2.6^{* *}$ \\
& & & \\
IVCT (ms) & $9.4 \pm 1.0$ & $9.6 \pm 0.6$ & $10.2 \pm 0.8$ \\
IVRT (ms) & $14.4 \pm 0.5$ & $17.4 \pm 0.4^{* * *}$ & $14.9 \pm 0.7^{\# \#}$ \\
\hline
\end{tabular}

LV, left ventricle; LVID;d, left ventricle internal diameter diastole; LVID;s, left ventricle internal diameter systole; RWT; d, relative wall thickness diastole; RWT;s, relative wall thickness systole; EDV, end-diastolic volume; ESV, end-systolic volume; SV, stroke volume, EF; ejection fraction; IVCT, isovolumic contraction time; IVRT, isovolumic relaxation time. Significantly different from WT, $* p<0.05, * * p<0.01$; significantly different from $m d x$-vehicle, ${ }^{\# \#} p<0.01$; using a One-way ANOVA with a Tukey's post-hoc test, (n=10-12 per group). 


\section{FIGURE LEGENDS}

Figure 1. GSK3 $\beta$ is more active in EDL muscles from D2 mice vs C57 mice, and tideglusib treatment inhibits GSK3b in D2 $\boldsymbol{m} \boldsymbol{d} \boldsymbol{x}$ mice. a) Representative Western blot images of GSK3 $\beta$ in EDL muscles from 9-10 week old D2 and C57 WT and $m d x$ mice. b) Total GSK3 $\beta$ analysis in EDL muscles from D2 and C57 WT and $m d x$ mice ( $\mathrm{n}=9-12$ per group). c) Ser9 phosphorylated GSK3 $\beta$ (relative to total) analysis in EDL muscles from D2 and C57 WT and $m d x$ mice ( $\mathrm{n}=9-12$ per group). d) Four weeks of tideglusib treatment lowers total GSK3 $\beta$ content and increases Ser9 phosphorylated GSK3 $\beta$ (relative to total) content in EDL muscles from D2 $m d x$ mice $(\mathrm{n}=5-6$ per group). e) Tideglusib significantly raises b-catenin content in EDL muscles from D2 $m d x$ mice (n $=6$ per group). For b and c, a two-way ANOVA was used with a Tukey's post-hoc test. For d and e, a Student's t-test was used, ${ }^{*} p<0.05$. All values are means \pm SEM.

Figure 2. Tideglusib treatment increases muscle mass, the percentage of oxidative fibers and utrophin expression while also reducing muscle necrosis, serum creatine kinase (CK) and oxidative stress. a) EDL:body mass ratio is significantly lowest in vehicle treated $m d x$ mice when compared with both WT and tideglusib treated $m d x$ mice ( $n=10-12$ per group). b) Fold-change body composition (body mass, lean mass and fat mass) analysis at baseline and after 4 weeks of tideglusib (or vehicle) treatment ( $n=10-12$ per group). c and d) Immunofluorescent fiber type compositional analysis in EDL muscle shows that tideglusib enhances the proportion of oxidative (type I and IIA) fibers ( $\mathrm{n}=4$ per group) (scale bars $=1000 \mathrm{~mm}$ ). Type I, immunostained blue; type IIA immunostained, green; type IIB, immunostained red; type IIX, unstained and appear as black. e) Utrophin $m R N A$ analysis in plantaris muscles from vehicle treated and tideglusib treated D2 $m d x$ mice ( $\mathrm{n}=7-10$ per group). f and g) Representative H\&E images revealing a significant reduction in muscle necrosis (areas occupied by fibrosis and immune cell infiltration) (n=4 per group). h) Serum CK is significantly elevated in vehicle treated $m d x$ mice compared with WT and tideglusib treated $m d x$ mice. i) Total nitrotyrosine and nitrocysteine western blot analysis in EDL muscles from WT, vehicle and tideglusib treated D2 $m d x$ mice $(\mathrm{n}=6$ per group). For $\mathrm{a}, \mathrm{b}, \mathrm{h}$ and $\mathrm{I}$, a one-way ANOVA with a Tukey's post-hoc test was used. For c-g, a Student's t-test was used. For all statistical analysis, ${ }^{*} p<0.05,{ }^{* *} p<0.01,{ }^{* * *} p<0.001,{ }^{* * * *} p<0.0001$. All values are means \pm SEM.

Figure 3. Whole-body and isolated muscle performance in WT, vehicle treated and tideglusib treated D2 $\boldsymbol{m} d \boldsymbol{x}$ mice. a) Daily and dark-period cage activity is significantly lower in vehicle treated $m d x$ mice vs WT mice, but cage activity in tideglusib treated D2 $m d x$ mice is not $(\mathrm{n}=6$ per group). b) Hangwire impulse is significantly lower in vehicle treated, but not tideglusib treated, $m d x$ mice vs WT mice ( $\mathrm{n}=10-12$ per group). c) Time to reach $50 \%$ of initial force after a fatigue protocol $(70 \mathrm{~Hz}$ every $2 \mathrm{~s}$ for $5 \mathrm{~min})$ is lower in vehicle-treated $m d x$ EDL muscles vs WT mice $(\mathrm{n}=$ 6-10 per group). d) Force-frequency curve analysis from isolated EDL muscles ( $\mathrm{n}=6-10$ per group). For a-c, a one-way ANOVA with a Tukey's post-hoc test was used. For d, a two-way ANOVA was used with a Tukey's post-hoc test. For a-c, ${ }^{*} p<0.05$ and ${ }^{*} p<0.01$. For $\mathrm{d},{ }^{*} p<0.05$,

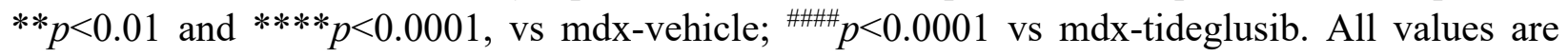
means \pm SEM. 
Figure 4. GSK3 $\beta$ inhibition improves cardiac SERCA function in tideglusib treated D2 $m d x$ mice. a-b) Expression of phosphorylated (pGSK $\beta$ ), total (GSK3 $\beta$ ) and the ratio of pGSK3 $\beta$ :GSK3 $\beta$ content quantified relative to vehicle using western blot analysis normalized to ponceau stain with a significant reduction in GSK3 $\beta$ in mdx-tideglusib vs mdx-vehicle treated mice ( $n=5-6$ per group). c) Representative $\mathrm{Ca}^{2+}$ uptake tracings over time initiated with ATP. d) Total $\mathrm{Ca}^{2+}$ uptake rate measured over a $2 \mathrm{~min}$ period and normalized to total protein is significantly reduced in both mdx-vehicle and tideglusib treated mice vs WT ( $\mathrm{n}=5-6$ per group). e) Maximal SERCA ATPase activity is significantly reduced in mdx-vehicle vs. WT mice. f) SERCA activity$p$ Ca curves in LV homogenates from WT, mdx-vehicle and mdx-tideglusib treated mice $(\mathrm{n}=5-6$ per group). g-h) Western blot analysis of SERCA2 and PLN expression normalized to GAPDH in mdx-vehicle and mdx-tideglusib treated mice relative to WT. i-j) Western blot analysis of total nitrotyrosine and nitrocysteine residues in LV homogenates normalized to ponceau stain in WT, mdx-vehicle and mdx-tideglusib treated mice ( $n=5-6$ per group). For $b, d, e, h$, and $j$, a one-way ANOVA with Tukey's post-hoc test was used. For b and j, ${ }^{*} p<0.05$ vs mdx-vehicle. For c-h, ${ }^{*} p<0.05$ and $* * * * p<0.0001$ vs WT. All values are means $\pm \mathrm{SEM}$.

Figure 5. LV fibrosis in vehicle treated and tideglusib treated D2 $m d x$ mice. a) Gamori trichrome stain images from the apex and base of WT, mdx-vehicle and mdx-tideglusib hearts. Red $=$ cytoplasm, black $=$ nuclei, blue $=$ collagen. $($ Scale bar $=100 \mu \mathrm{m}) \mathbf{b})$ Histological analysis of $\%$ fibrosis of the apex and base of WT, mdx-vehicle and mdx-tideglusib hearts. Two-way ANOVA was used with Welch's correction for unequal variance to analyze \% fibrosis with a trending main effect of treatment ( $p=0.12 ; \mathrm{n}=5$ per group). All values are means $\pm \mathrm{SEM}$. 


\section{Online Methods}

Animals

All mice, C57 mdx (stock \#013141), D2-mdx (stock \#001801), C57 WT (stock number 000476) and D2 WT (stock \#000671), were ordered from Jackson Laboratories. Upon arrival, they were acclimated and housed in Brock University's Animal Facility, in an environmentally controlled room with a standard 12:12 hour light-dark cycle and allowed access to food and water ad libitum. All animal procedures were approved by the Brock University Animal Care and Utilization Committee (file \#17-06-03) and were carried out in accordance with the Canadian Council on Animal Care guidelines.

\section{Tideglusib treatment}

D2 $m d x$ mice were treated with tideglusib at a dose of $10 \mathrm{mg} / \mathrm{kg} /$ day via oral gavage, whereas a separate vehicle group of D2 $m d x$ mice was given $26 \%$ peg $400,15 \%$ Chremaphor EL and water ${ }^{11}$ for 5 days. A separate group of WT mice were also housed simultaneously to serve as a healthy reference control. D2 $m d x$ mice were treated for a period of 2-4 weeks to allow for a variety of in vivo measures to be conducted. A subset of D2 $m d x$ (vehicle and tideglusib) mice were euthanized after 2 weeks to examine GSK3 activation, fiber type composition and necrosis via histological staining ( $n=5-6$ per group). The remaining mice were treated for an additional two weeks to conduct several in vivo measures and additional biochemical (SERCA function and western blotting) and histological measures (cardiac histology) ( $\mathrm{n}=10-12$ per group).

\section{Body composition}

A small animal DXA scanner (OsteoSys InSIGHT, Scintica) was used to non-invasively measure body composition in anesthetized mice (vapourized isoflurane $5 \%$ in $\mathrm{O}_{2}$ ) at baseline and 
after 4 weeks of tideglusib or vehicle treatment. Absolute quantities of body, lean and fat mass (g) were obtained.

\section{Whole-body muscle performance}

Whole-body muscle performance was first measured immediately after 2 weeks of tideglusib treatment using a hangwire tests according to the Neuromuscular Disease Network Standard Operating Procedure: DMD_M.2.1.004. All mice were gently placed on the wire situated 12 inches high and were left suspended on the wire until they reached exhaustion and dropped from the wire to the base of the cage. The time they remained suspended was recorded for three trials, separated by a 60 s recovery period, and impulse $\left(\mathrm{s}^{*} \mathrm{~g}\right)$ was calculated by multiplying the average time suspended by body mass. Secondly and after 3 weeks of treatment, mice were placed in a Promethion Metabolic Cage System for 48 hours (two 12-hour light and dark cycles) to measure cage activity. Cage ambulation was quantified through meters travelled which is collected through beam breaks with the BXYZ Beambreak Activity Monitor in the $\mathrm{x} y$, and z planes.

\section{High frequency ultrasound}

On the 4th week of tideglusib or vehicle treatment, D2 $m d x$ and healthy WT mice underwent high frequency ultrasound analysis using the Prospect T1 high frequency ultrasound (S-Sharp, Taiwan). Short axis M-mode imaging was used to calculate systolic function, including the LVID, EDV, and ESV. While PW Doppler of the mitral valve inflow, from an apical 4-chamber view, was used to assess diastolic function, specifically measuring the IVRT/CT. Measurements were taken at a maintained heart rate of 400-500 bpm. 


\section{Sample and tissue collection}

After the C57 and D2 mice reached 9-10 weeks of age, and after D2 $m d x$ mice underwent 2 or 4 weeks of tideglusib (or vehicle) treatment, they were euthanized via cervical dislocation under general anesthetic (vaporized isoflurane) and their tissues were collected. Blood was collected via cardiac puncture and spun at $5000 \mathrm{xg}$ for 8 minutes $\left(4^{\circ} \mathrm{C}\right)$ and serum was collected and stored at $-80^{\circ} \mathrm{C}$. Extensor digitorum longus (EDL), plantaris, diaphragm and cardiac muscles were isolated for further analysis.

\section{Isolated muscle contractility}

After 4 weeks of tideglusib or vehicle treatment, EDL muscles from D2 $m d x$ and D2 WT mice were carefully dissected and mounted onto an Aurora Scientific contractile apparatus (model 305B $\& 701 \mathrm{~B})$ to assess muscle force production and fatigue as previously described ${ }^{61}$. Muscles were stimulated using a biphasic stimulator (Model 701B, Aurora Scientific, Inc.) with all data sampled at $1 \mathrm{kHz}$ and saved to computer for further analysis (ASI 600a software). EDL muscles were subjected to a force-frequency $(1-150 \mathrm{~Hz})$ and fatigue protocol comprising a $70 \mathrm{~Hz}$ volley every $2 \mathrm{~s}$ for $5 \mathrm{~min}$ with a sampling rate of $2000 \mathrm{~Hz}$. For data analyses, peak isometric force amplitude $(\mathrm{mN})$ was determined across the range of stimulation frequencies and throughout the fatigue protocol. Peak isometric force was then normalized to muscle cross-sectional area (CSA), which was calculated using the following formula: CSA $=m / 1 * d^{*}\left(L_{f} / L_{o}\right)$, where $m$, muscle mass (mg); 1 , muscle length (mm); d, mammalian skeletal muscle density $\left(1.06 \mathrm{mg} / \mathrm{mm}^{3}\right)^{62} \mathrm{Lf} / \mathrm{Lo}$ is the fiber length-to-muscle length ratio $(0.44 \text { for the } \mathrm{EDL})^{63}$. For analyses of fatigue, the time required to reach $50 \%$ of initial force was recorded ${ }^{64}$. 


\section{Serum CK analysis}

Serum creatine kinase (CK) activity was measured with a M2 Molecular Device plate reader and a commercially available assay (Cat. \#C7522, Pointe Scientific Inc., Canton, MI, USA) fitted onto a 96-well plate and calibrated with a standard curve of purified creatine kinase (Sigma, Oakville, ON, Canada, Cat. 10127566001).

\section{Immunofluorescent fiber typing}

Fiber typing was accomplished through via immunofluorescent staining of myosin heavy chain (MHC) isoforms in EDL muscles collected from D2 $m d x$ mice treated with tideglusib or vehicle for two weeks as previously described ${ }^{65}$. Briefly, EDL muscles frozen in O.C.T. compound were sliced into $10 \mu \mathrm{m}$ sections using a HM525NX Cryostat (ThermoFisher Scientific, MI, USA) and placed onto vectabonded microscope slides. Slides were then blocked with $10 \%$ goat serum (5425, Cell Signaling; in phosphate-buffer solution (PBS)) prior to incubation with MHC antibodies targeting MHCI (1:50; BA-F8), MHCIIa (1:600; SC-71), and MHCIIb (1:100; BF-F3) (obtained from the Developmental Studies Hybridoma Bank (University of Iowa, Iowa, City, IA). A cocktail of secondary antibodies which included: Alexa Fluor 350 (IgG2b for MHCI; A-21140 ThermoFisher Scientific), Alexa Fluor 488 (IgG1 for MHCIIa; A-21121 ThermoFisher Scientific), and Alexa Fluor 555 (IgM for MHCIIb, A-21426 ThermoFisher Scientific,MI, USA) were used to visualize the various fiber types. Slides were imaged using a BioTek Cytation 5 Multimode Plate Reader at 10x magnification using auto-exposure settings for three filters: DAPI, GFP, and Texas Red. Images were then stitched, processed and saved using the Gen5 image processing functions, and then analyzed using imageJ (NIH) software to estimate fiber type composition. 


\section{Histological assessment}

For EDL muscles, necrosis (was measured using Hematoxylin and Eosin (H\&E) staining of EDL muscle samples. All slides were imaged using a BioTek Cytation 5 Multimode Plate Reader. All images were acquired using 10x magnification using auto-exposure settings for the Colour Brightfield setting. Images were then saved and then analyzed using imageJ (NIH) software to estimate necrotic area. This was done by calculating the total surface area of the muscle, then calculating the sum of the area of all necrosis (areas occupied by collagen and immune cell infiltration) within the sample. This total necrosis was then divided by the total area of the sample.

For cardiac histology, hearts were isolated and fixed in $10 \%$ formalin for $72 \mathrm{hrs}$ then transferred to $70 \%(\mathrm{v} / \mathrm{v})$ ethanol until being embedded in paraffin wax ( $\mathrm{n}=5$ per group). Slices 10 $\mu \mathrm{m}$ in size were affixed to vectabond-treated slides and stained with Gamori trichrome stain with muscle tissues stained red, nuclei stained black and fibrotic tissue stained blue. Images were taken at 20x magnification at the apex and base of the heart (4-6 images per area). Images were analyzed using ImageJ software (US National Institutes of Health) to identify \% fibrosis by measuring total blue area (fibrotic tissue) divided by total red area (muscle tissue) x $100 \%{ }^{66}$.

\section{Western blotting}

Western blotting was done to assess protein levels of GSK3 $\beta$, Ser9 phosphorylated GSK3 $\beta, \beta$-catenin, SERCA1, SERCA2, PLN, and total protein nitration (nitrotyrosine) and nitrosylation (nitrocysteine). Standard SDS-PAGE was performed with BioRad 7-12\% TGX gels (4561086; BioRad, Hercules, CA, USA) and polyvinylidine difluoride (PVDF) membranes were used for all proteins (BioRad). All protein blots were blocked with Everyblot buffer (\#12010020, BioRad) for $10 \mathrm{~min}$ at room temperature and incubated with primary and corresponding secondary 
antibodies (Supplementary Table 1) prior to imaging with a BioRad ChemiDoc Imager with Immobilon ECL Ultra Western HRP Substrate (WBKLS0500, Sigma-Aldrich). Images were analyzed using BioRad ImageLab software (BioRad) and were normalized to total protein analyzed on a Ponceau stain or GAPDH ${ }^{66,67}$.

$m R N A$ analysis

$m R N A$ analyses for utrophin (forward, GGGGAAGATGTGAGAGATTT; reverse, GTGTGGTGAGGAGATACGAT) was accomplished by homogenizing plantaris samples from vehicle treated and tideglusib treated D2 $m d x$ mice ( $\mathrm{n}=7-10$ per group) with $1 \mathrm{~mL}$ of TRIzol. A commercially available Qiagen RNeasy kit (Quiagen, Hilden, Germany, ID \#74104) and a DNase Max kit (Quiagen, Hilden, Germany, D \#15200-50) was then used to isolate RNA. This was then quantified using a NanoVue Plus spectrophotometer (Biochrom Ltd., Cambridge, UK), followed by the use of EcoDry RNA to cDNA (Takara Bio Inc., Kusatsu, Shiga, Japan, ID \#639547) reaction tubes and a SimpliAmp Thermal Cylinder (ThermoFisher, MA, USA, ID \#A24811) to facilitate the generation of cDNA. Diluted cDNA was then analyzed using a qPCR reaction 96-well plate and the StepOnePlus Real-Time PCR System (ThermoFisher, MA, USA, ID \#4376600). Threshold cycle $(\mathrm{Ct})$ values were recorded, and data were analyzed using the $\Delta \Delta \mathrm{Ct}$ method and reported as a foldchange $(2 \Delta \mathrm{Ct})$, using expression of the Gapdh housekeeping gene as a reference (forward, CGGTGCTGAGTATGTCGTGGAGTC; reverse, GGGGCTAAGCAGTTGGTGGTG; IDT) 


\section{SERCA function}

SERCA function was assessed in diaphragm and cardiac muscle homogenates using an oxalate-supported $\mathrm{Ca}^{2+}$ uptake fluorimetric assay and an ionophore (A23187, Sigma Aldrich)supported spectrophotometric SERCA activity assay, both fitted onto a 96-well plate using an M2 MultiMode plate reader (Molecular Devices) ${ }^{68}$. Calcium uptake in muscle homogenates was measured using the Indo-1 $\mathrm{Ca}^{2+}$ fluorophore-dependent (57160, Sigma-Aldrich), which is a ratioable dye can provide an indication of free cytosolic $\mathrm{Ca}^{2+}$ levels when excited at $355 \mathrm{~nm}$ and emitted at $405 \mathrm{~nm}\left(\mathrm{Ca}^{2+}\right.$-bound Indo-1) and 485nm (free Indo-1) using the Kd of $250 \mathrm{nM}$. SERCA specific activity in muscle homogenates was determined using an enzyme-linked spectrophotometric assay that measures ATP hydrolysis indirectly through NADH disappearance (kinetic absorbance at $340 \mathrm{~nm}$ ) with and without the SERCA-specific inhibitor cyclopiazonic acid (CPA). SERCA activity was measured at a varying $\mathrm{Ca}^{2+}$ concentrations ranging from $\mathrm{pCa}$ 5-7. Activity- $p$ Ca curves were fitted onto a sigmoidal dose response curve (variable slope) on Graphpad Prism 8 software (GraphPad Software Inc.) ${ }^{66,67}$ to obtain the $p \mathrm{Ca}_{50}$ - the $\mathrm{Ca}^{2+}$ concentration required to elicit $1 / 2$ Vmax. Maximal SERCA activity was taken from the raw data. For both $\mathrm{Ca}^{2+}$ uptake and SERCA activity, rates were normalized to total protein obtained from a BCA assay.

\section{Statistical analysis}

Statistical comparisons were made using a Student's t-test, a one-way or two-way ANOVA with a Tukey's post-hoc test where appropriate. All statistical analysis were done using Graphpad Prism 8 software. A Welch's t-test or ANOVA were used in cases where variability between groups significantly differed. Statistical significance was set to a $p \leq 0.05$ and a statistical trend set to $p \leq 0.15$. All values are presented as means \pm SEM. 


\section{Figure 1}
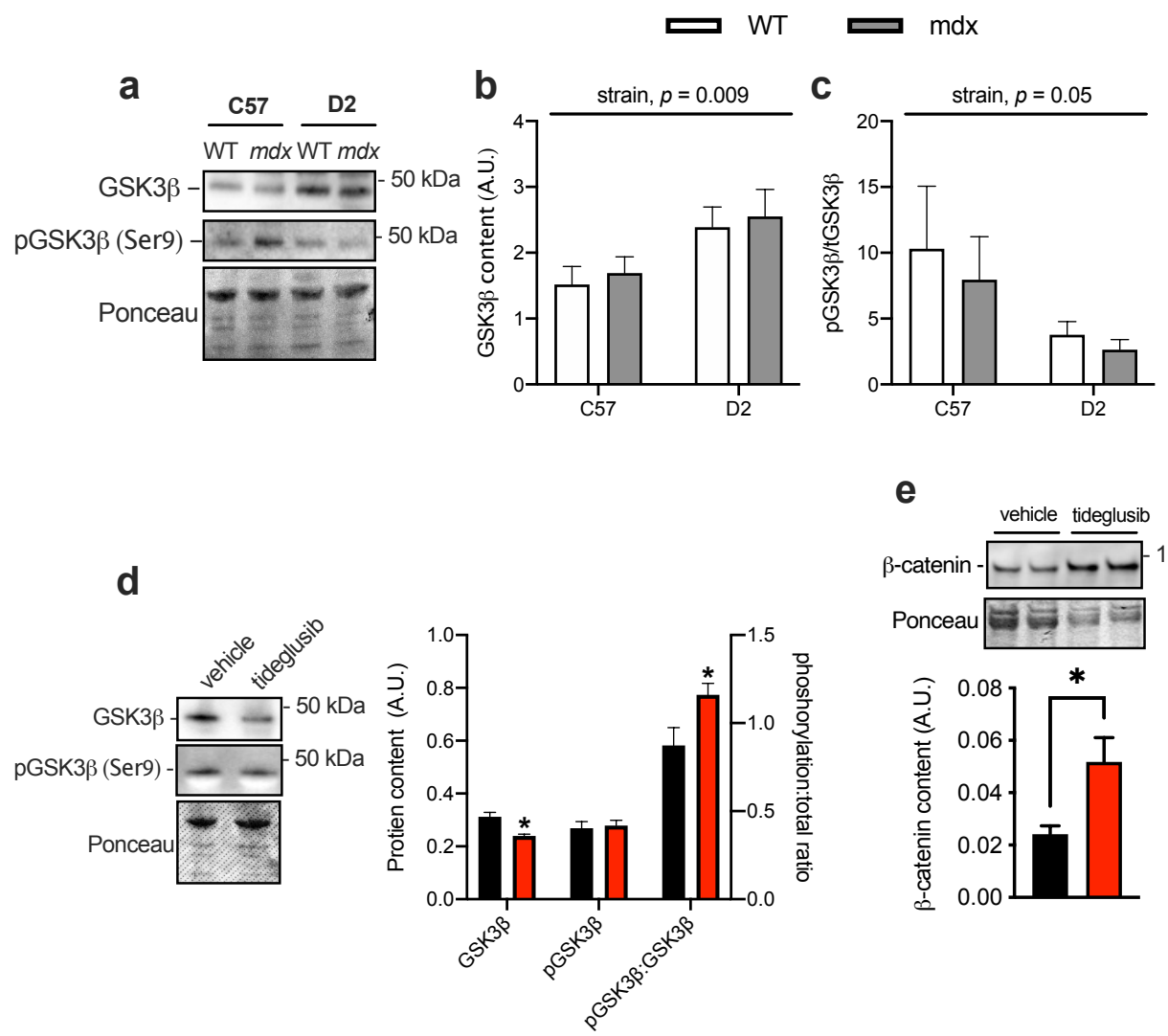

e

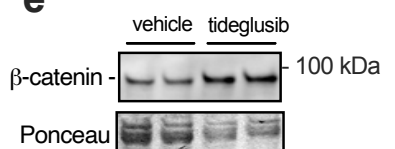

mdx-vehicle

mdx-tideglusib 


\section{Figure 2}

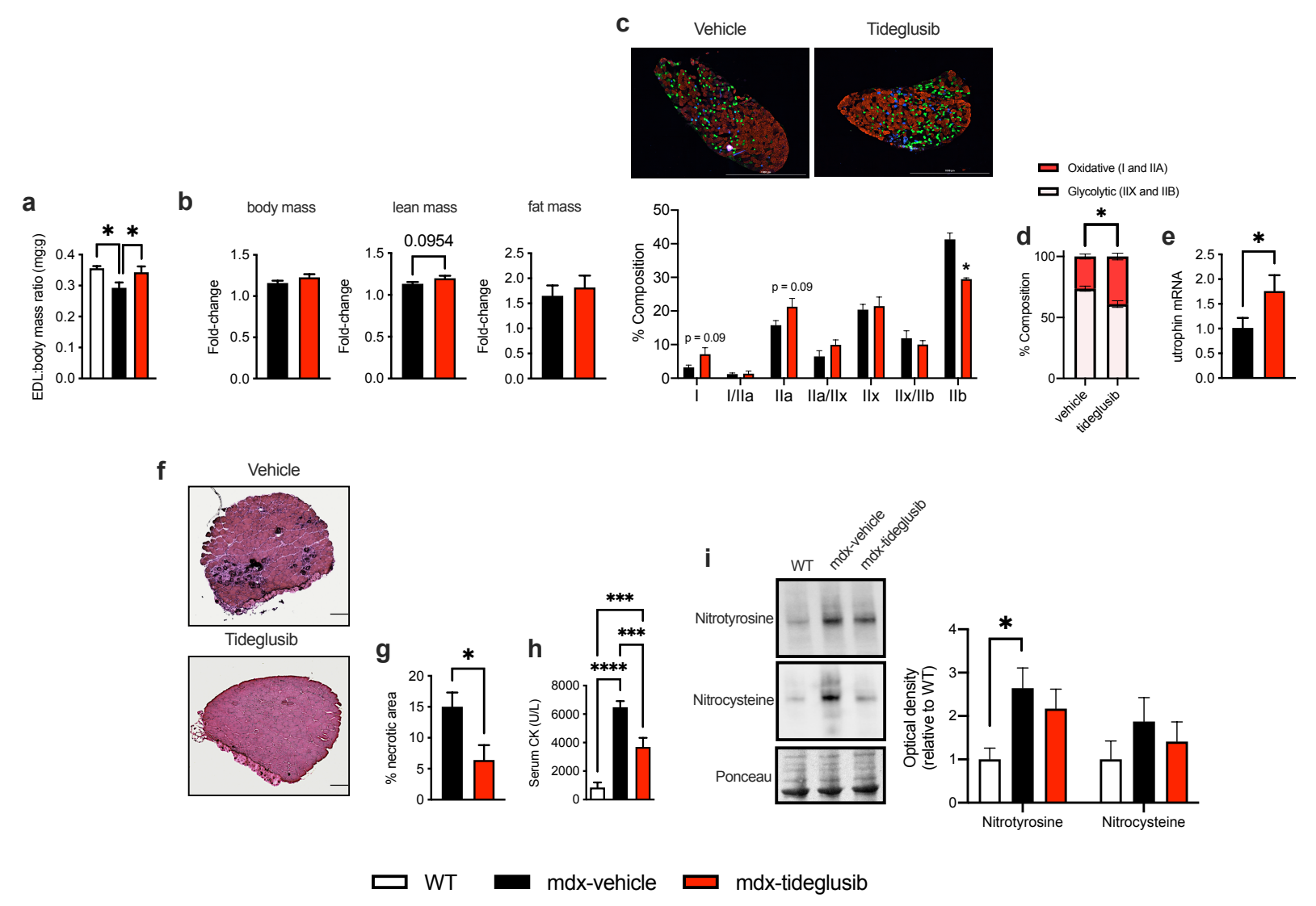




\section{Figure 3}

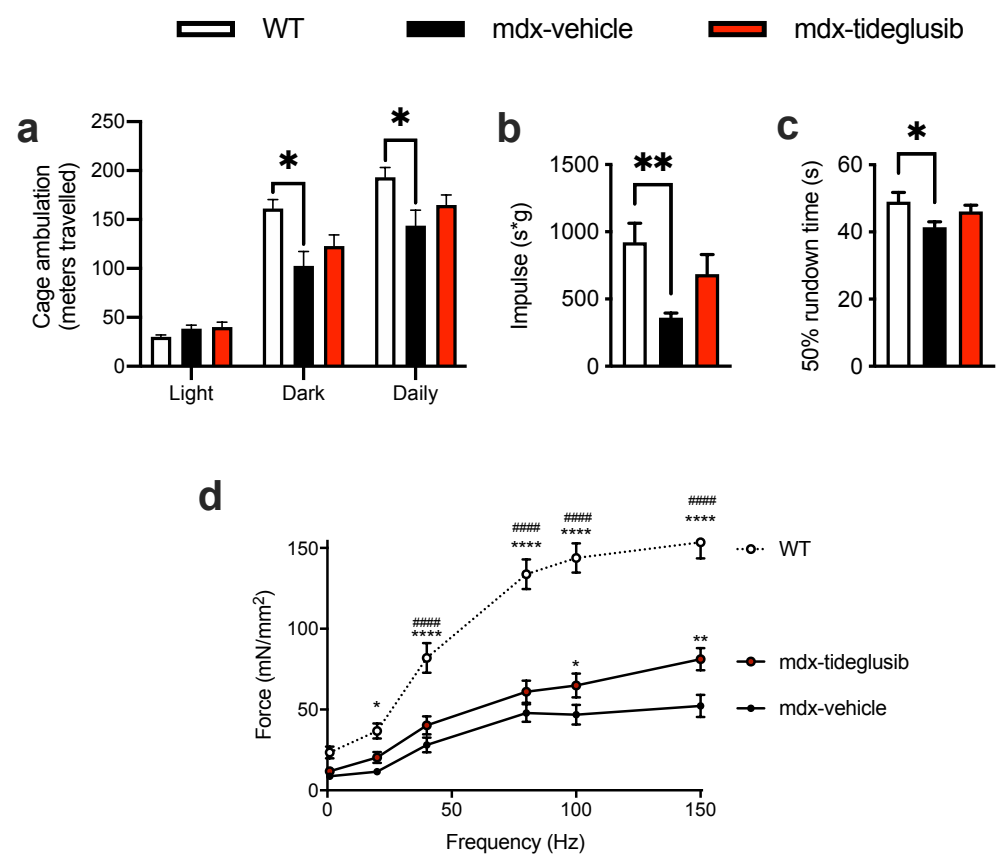




\section{Figure 4}
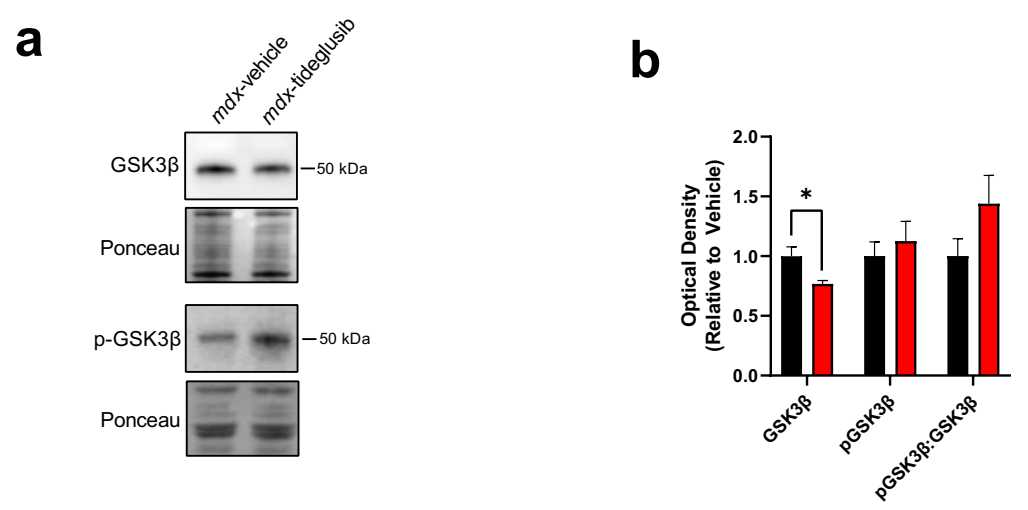

C

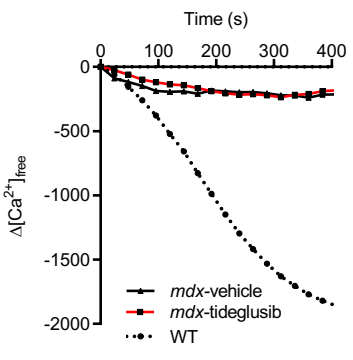

g

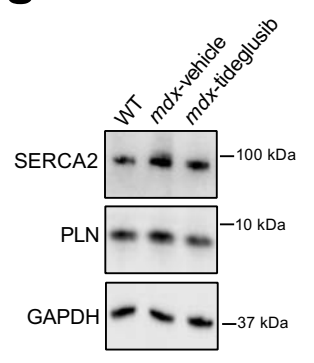

d

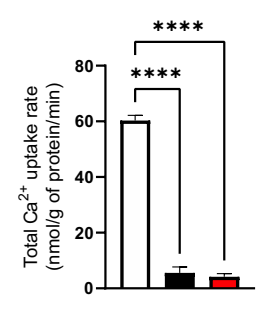

h

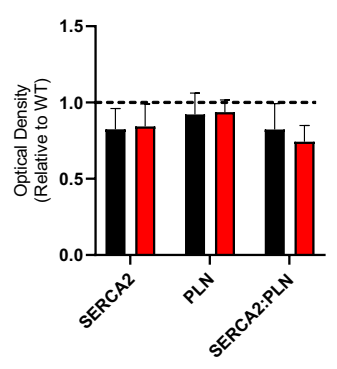

f

e
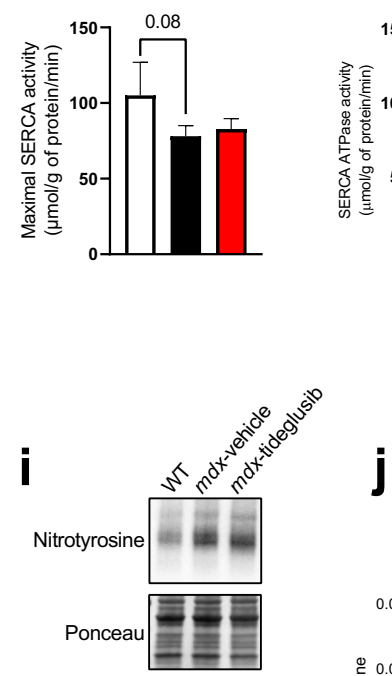

Nitrocysteine

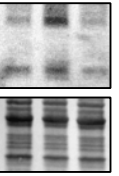

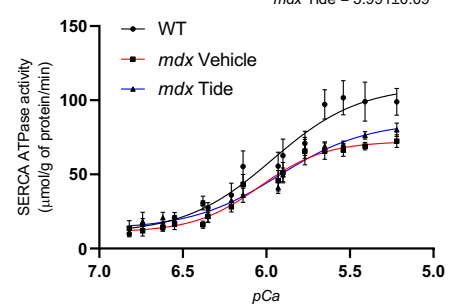
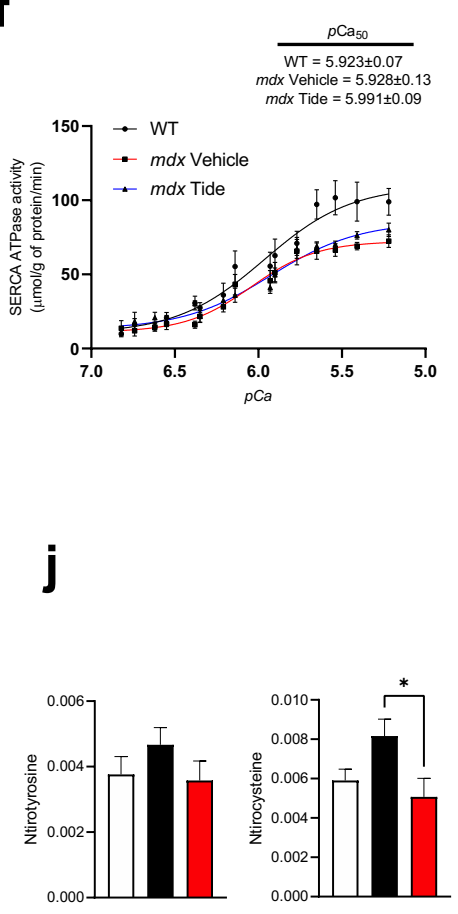

$\frac{1}{W T=5.923 \pm 0.07}$
$m d x$ Vehicle $=5.928 \pm 0.13$
$m d x$ Tide $=5.991 \pm 0.09$

j$$
0.000
$$

mdx-vehicle 


\section{Figure 5}

a

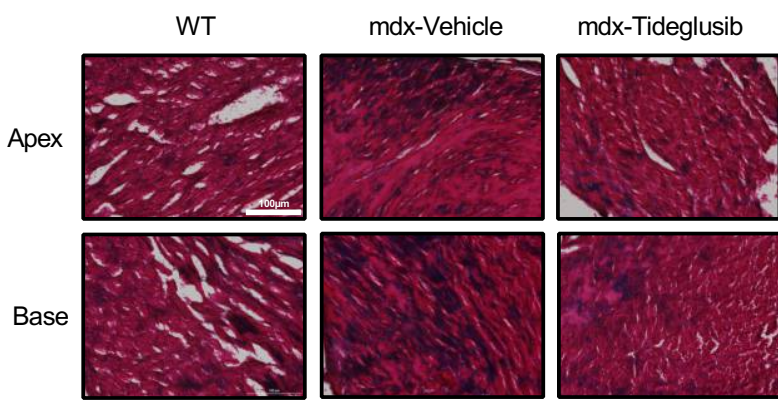

ש WT b

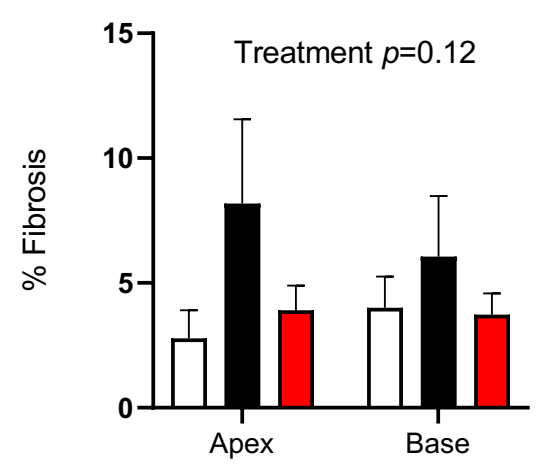

mdx-tideglusib 